\title{
Delayed remission following sequential infusion of humanized CD19- and CD22-modified CAR-T cells in a patient with relapsed/refractory acute lymphoblastic leukemia and prior exposure to murine-derived CDI9-directed CAR-T cells
}

This article was published in the following Dove Medical Press journal: OncoTargets and Therapy

Fei Yang $1, *$

Jian Zhangl,*

Xinyou Zhang ${ }^{2, *}$

Mengli Tian'

Jingjing Wang'

Liqing Kang ${ }^{3}$

Huiying Qiu'

Depei $\mathrm{Wu}^{\prime}$

'Department of Hematology, Jiangsu Institute of Hematology,

The First Affiliated Hospital of

Soochow University, Suzhou, People's

Republic of China; ${ }^{2}$ Department of

Hematology, The Second Clinical

Medical College of Jinan University

(Shenzhen People's Hospital), Jinan

University, Shenzhen, Guangdong

Province, People's Republic of China;

${ }^{3}$ Institute of Biomedical Engineering

and Technology, Shanghai Engineering

Research Center of Molecular

Therapeutics and New Drug

Development, School of Chemistry

and Molecular Engineering, East China

Normal University, Shanghai, People's

Republic of China

*These authors contributed equally to this work

Correspondence: Huiying Qiu

Department of Hematology, Jiangsu Institute of Hematology, The First

Affiliated Hospital of Soochow University,

No I88, Shizi Street, Suzhou, People's

Republic of China

$\mathrm{Tel} / \mathrm{fax}+865$ I 26778 I856

Email pursueyf08@।26.com
Background: CD19-modified CAR-T cells greatly influence responses in patients with relapsed/ refractory acute lymphoblastic leukemia (ALL). However, recurrence remains a challenge, and reinfusion of CAR-T cells is not always effective. Sequential infusion of humanized CD19modified and CD22-modified CAR-T cells may overcome this issue and induce remission.

Methods: We examined treatment with sequential infusion of humanized CD19-modified and CD22-modified CAR-T cells in a patient with relapsed ALL previously exposed to murinederived anti-CD19 CAR-T cells.

Results: At $\sim 6$ weeks after treatment, repeated bone marrow smear and flow cytometry analysis revealed no lymphoblasts.

Conclusion: Our results suggest that sequential infusion of humanized CD19-modified and CD22-modified CAR-T cells is a valuable option for relapsed patients with prior infusion of murine-derived, CD19-directed CAR-T cells.

Keywords: chimeric antigen receptor, anti-CD19, anti-CD22, humanized, acute lymphoblastic leukemia, relapsed

\section{Introduction}

Acute lymphoblastic leukemia (ALL) is the most common hematological malignancy occurring in childhood. The 5-year overall survival of pediatric ALL cases is $80 \%-90 \% .{ }^{1}$ However, the outcomes of approximately $15 \%-20 \%$ patients who relapse show a 5 -year overall survival of $<20 \%{ }^{2,3}$ Anti-CD19 CAR-T cells can produce potent and sustained responses in children with relapsed/refractory ALL. ${ }^{3-9}$ However, some patients show limited persistence, which can limit the effectiveness of anti-CD19 CAR-T cells and may increase the risk of relapse. One common reason for this effect is that most single-chain variable fragment ( $\mathrm{scFv}$ ) domains of the CAR structure are of murine origin, and repeated infusion would be ineffective in patients who show rapid CAR cell loss mediated by immune rejection; ${ }^{9}$ therefore, immune-mediated antimouse reactivity is a potential cause that may be corrected by fully humanized CAR designs. Additionally, CD22 is expressed in most cases of B-ALL and may be another suitable target for CAR-T therapy. CD22 CAR-T cells show similar safety profiles as CD19 CAR-T cells and can mediate antileukemia effects in CD19-weakly positive 
or CD19-negative patients relapsed after CD19-targeted immunotherapy. ${ }^{10}$ We developed CAR-T cells harboring a humanized anti-CD19 scFv structure and another group of CAR-T cells with a humanized anti-CD22 scFv composition. We conducted treatment by sequential infusion of humanized CD19-modified and CD22-modified CAR-T cells in a patient with relapsed ALL previously exposed to murine-derived, CD19-targeted CAR-T cells.

\section{Patients and methods}

\section{Patients}

The patient was a 9-year-old girl who was treated at the First Affiliated Hospital of Soochow University in China. She presented with fever and muscle and joint pain on November 20, 2016. Blood examination revealed a white blood cell count of $28.99 \times 10^{9} / \mathrm{L}$, hemoglobin level of $119 \mathrm{~g} / \mathrm{L}$, and platelet count of $24 \times 10^{9} / \mathrm{L}$. At presentation, her physical examination showed splenomegaly and bilateral foot dorsal skin petechiae. The bone marrow (BM) smear showed $88.5 \%$ blasts. Flow cytometry analysis showed 97.6\% CD10+ and 97.7\% CD19+. She had a normal female karyotype, and a KRAS T58I mutation without fusion genes was detected. ALL was then diagnosed. She was administered chemotherapy with the cyclophosphamide, vindesine, daunorubicin, and dexamethasone protocol for 10 days post diagnosis. Symptoms were alleviated, and she achieved complete remission at 30 days post diagnosis.

The patient suffered from sore throat and fever, and repeated BM aspirates revealed 51\% lymphoblasts at 44 days post diagnosis in 2017. She was administered the hypercyclophosphamide, vindesine, daunorubicin, and dexamethasone protocol as reinduction chemotherapy. BM revealed no lymphoblasts and a minimal residual disease

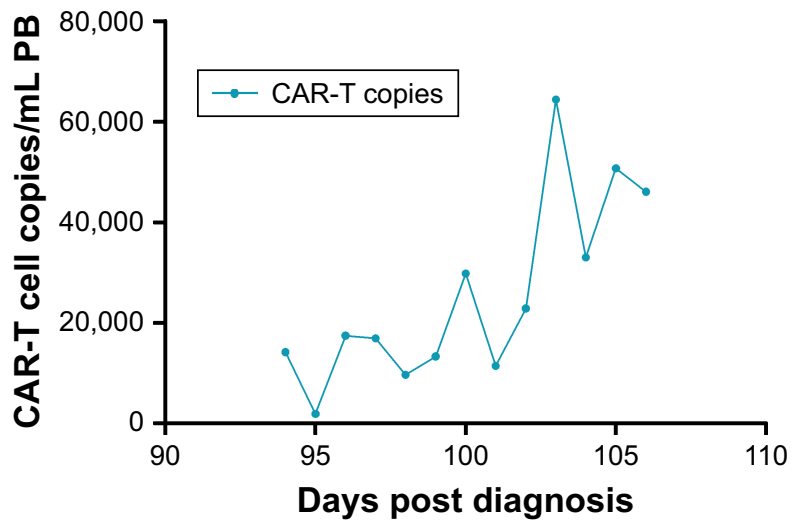

value of $0.1 \times 10^{-4}$ at 65 days post diagnosis. However, the patient underwent disease recurrence again at 80 days post diagnosis. BM aspirates showed 76.5\% lymphoblasts. Flow cytometry analysis revealed $87.8 \% \mathrm{CD} 10+$ and $97.0 \%$ CD19+. Subsequently, $150 \mathrm{~mL}$ peripheral blood (PB) samples were used to cultivate anti-CD19 CAR-T cells. Lymphodepleting chemotherapy with the $\mathrm{FC}$ regimen (cyclophosphamide $300 \mathrm{mg} / \mathrm{kg}$, fludarabine $30 \mathrm{mg} / \mathrm{m}^{2}$, days -9 to -7 ) was administered at 83 days post diagnosis. Next, she was infused with CD19-directed CAR-T cells that had been amplified with anti-CD3 and 4-1BB antibodies and lentivirally transduced to express the anti-CD19 CARs (The Unicar-Therapy Bio-medicine Technology Co., Ltd., Shanghai, China). The total dose was $5 \times 10^{6} \mathrm{CAR}$-positive $\mathrm{T}$ cells/kg (transduction efficiency was $50.66 \%$ ), administered over a period of 2 consecutive days on days 92 and 93 post diagnosis. She developed a high body temperature on day 1 after infusion of CAR-T cells and underwent hypotension requiring continuous vasopressors on day 3 and hypoxia requiring continuous nasal catheter oxygen inhalation on day 4. Additionally, a series of PB cytokines including IL-2, IL-4, IL-6, IL-10, tumor necrosis factor, interferon- $\gamma$, and IL-17A were detected. Of the seven types of cytokines detected, IL-6 showed the greatest increase, with a maximum fold change of 700 on day 4 . Next, severe cytokine release syndrome was diagnosed. The patient showed increased cytokine levels, anti-CD19 CAR-T cells, indicating grade I cytokine release syndrome (Figure 1). The patient's body temperature decreased to a normal value after treatment with nonsteroidal drugs. The IL-6 receptor-blocking monoclonal antibody tocilizumab $(8 \mathrm{mg} / \mathrm{kg})$ was prescribed combined with methylprednisolone $(2 \mathrm{mg} / \mathrm{kg}$ ) on day 4 . The symptoms were alleviated, and PB cytokines gradually decreased. BM

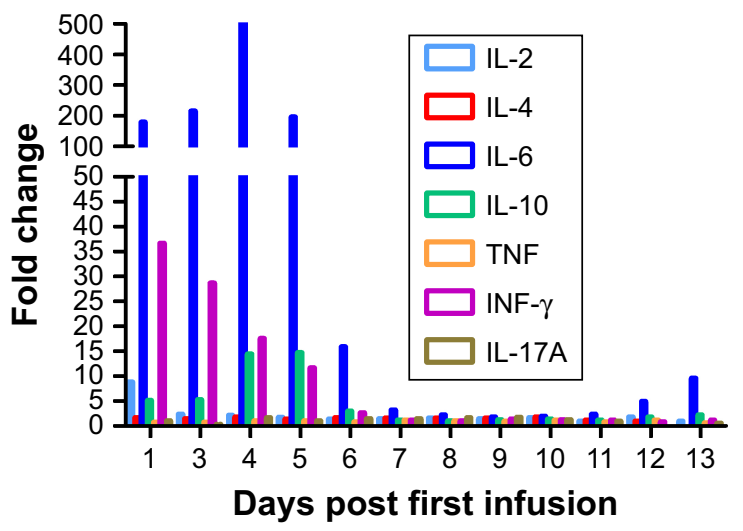

Figure I PB CAR-T cell copies and serum cytokine levels increased after first infusion of anti-CDI9 CAR-T cells. Abbreviation: PB, peripheral blood. 


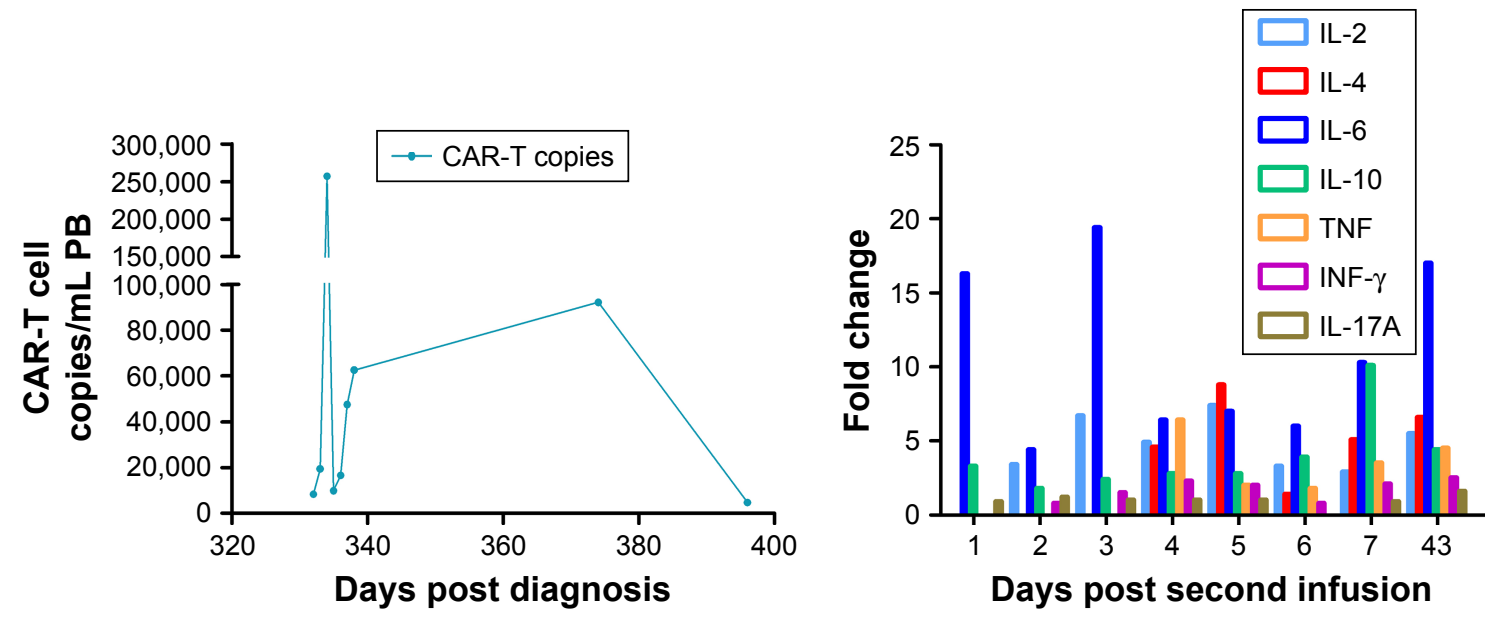

Figure 2 PB CAR-T cell copies and serum cytokine levels increased after sequential infusion of CAR-T cells. Abbreviation: PB, peripheral blood.

smear revealed 3\% lymphoblasts and a minimal residual disease of $0.0 \times 10^{-4}$ at 100 days post diagnosis. Changes in the levels of PB CAR-T cell copies and serum cytokines are summarized in Figure 1.

Allogeneic hematopoietic stem cell transplantation was suggested but refused. Unfortunately, the third recurrence occurred at 180 days post diagnosis. Repeated BM aspirates showed $25 \%$ lymphoblasts. Two cycles of reinduction chemotherapy were administered consisting of vindesine, daunorubicin, dexamethasone, and methotrexate. Unfortunately, the patient failed to achieve the fourth remission. BM smear showed $83.5 \%$ lymphoblasts at 302 days post diagnosis. Flow cytometry analysis revealed $98.9 \%$ CD10+, 98.8\% CD19+, and 9.9\% CD22+ cells. She was administered a second infusion of CAR-T cells intermittently following the FC regimen, which contained two different groups of CAR-T cells harboring the humanized scFv domain targeting CD19 and CD22. The total dose of humanized CD19directed CAR-T cells (transduction efficiency was $25 \%$ ) and humanized anti-CD22 CAR-T cells (transduction efficiency was $32 \%$ ) was the same as $5 \times 10^{6} \mathrm{CAR}$-positive $\mathrm{T}$ cells $/ \mathrm{kg}$, administered over a period of 2 consecutive days (days 326 and 327 post diagnosis) for anti-CD22 CAR-T cells and 2 consecutive days (days 331 and 332 post diagnosis) for CD19-targeted CAR-T cells, respectively. She developed a moderate body temperature, which was controlled by nonsteroidal anti-inflammatory drugs. The increase in the IL-6 level was lower than the previously observed increase, with a maximum fold change of 36 after infusion of CAR-T cells. Approximately 6 weeks later, on day 374 post diagnosis, repeated BM smear and flow cytometry analysis revealed no lymphoblasts. Additionally, increased PB CAR-T copies and cytokines were detected. Changes in the levels of PB CAR-T cell copies and serum cytokines are summarized in Figure 2. Changes in blasts, CD19+ cells, and CD22+ cells in the BM are summarized in Figure 3. Changes in BM lymphoblasts are summarized in Figure 3. She was in complete remission until January 1, 2018 (398 days post diagnosis).

\section{Methods}

Mouse-derived CAR-19

We designed a modified CAR construct containing an FMC63-derived CD19-specific scFv, CD3 $\zeta$ signaling domain, and 4-1BB costimulatory domain. ${ }^{11} \mathrm{CD} 3+\mathrm{T}$ cells collected from patients were activated with $\mathrm{CD} 3 / \mathrm{CD} 28$

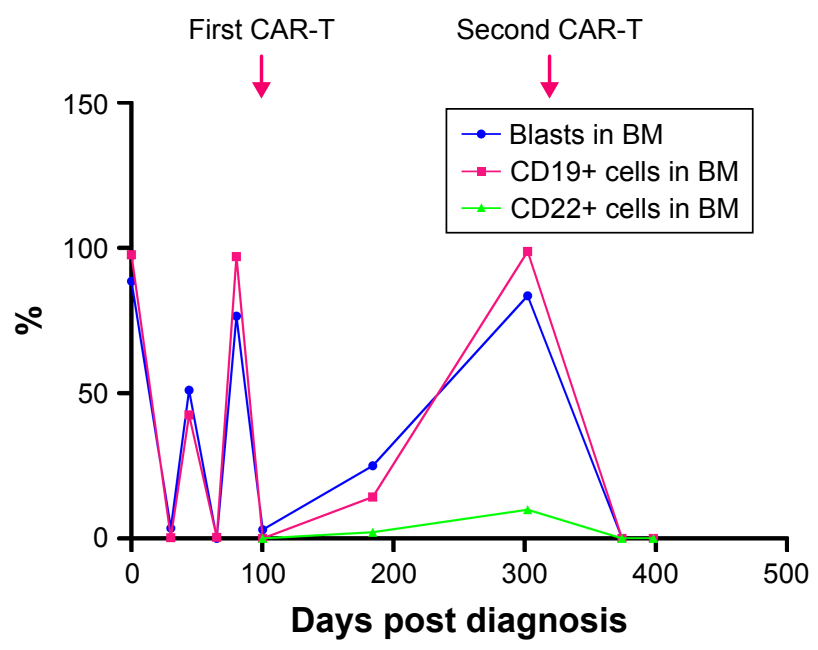

Figure 3 Changes in BM lymphoblasts and CAR-T therapy time. Abbreviation: BM, bone marrow. 
monoclonal antibodies (MACS, Teterow, Germany) at day 0 and lentivirally transduced at day 1 and then cultured in the X-VIVO 15 medium (Lonza Group, Ltd., Basel, Switzerland) containing $100 \mathrm{U} / \mathrm{mL}$ IL-2. The entire manufacturing procedure was conducted in the GMP Laboratory of Shanghai Unicar-Therapy Bio-medicine Technology, Co., Ltd. (Shanghai, China).

\section{Humanized CAR-19 and humanized CAR-CD22}

Humanized CAR-19 and humanized CAR-CD22 have the same costimulatory domain and co-activated domain as mouse-derived CAR-19, except for in the scFv of CAR; the humanized CAR-19 and CAR-CD22 recognition site is HD37.

\section{Measurement of CAR-T cells in vivo}

Genomic DNA was isolated directly from the PB of patients using a QIAamp DNA Blood Mini Kit (Qiagen NV, Venlo, the Netherlands). Quantitative real-time PCR was performed in triplicate using the ABI 2 TaqMan Universal Master Mix in a 7500 real-time PCR system (Thermo Fisher Scientific, Waltham, MA, USA) with AmpErase uracil-N-glycosylase (Thermo Fisher Scientific).

To determine the copy number per unit DNA, we set up a six-point standard curve consisting of 10 -fold serial dilutions of purified CAR plasmid containing $10^{-4}$ to $10^{-9}$ copies $/ \mathrm{mL}$. Primers/probes specific for the anti-CD19 CAR transgene and an internal control gene were used as described previously.

\section{Cell manufacture}

Patient T cells were cultured with an anti-CD3 monoclonal antibody and anti-CD28 monoclonal antibody to stimulate T-cell proliferation. The cells were transduced with recombinant lentiviral vectors containing the CD19-modified or CD22-modified scFv, cytoplasmic region of the 4-1BB costimulatory structure, and CD3z T-cell activation composition. This is the second-generation CAR applied in the clinic.

We generally set the multiplicity of infection to 5-10 for lentivirus transduction to ensure a transduction efficiency of $>20 \%$. After 48 hours of lentiviral transduction, the cells were expanded in vitro with X-Vivo 15 medium containing IL-7 and IL-15 cytokines for 7-10 days. The lentiviral transduction efficiency, ratio of CD4/CD8 T cells, T-cell differentiation degree, and cytotoxicity and cytokines release ability in vitro were evaluated.

\section{Persistence of CAR-T cells in patients}

Whole blood after CD19-modified or CD22-modified CAR-T cell infusion was collected in K2EDTA BD Vacutainer tubes
(BD Biosciences, San Jose, CA, USA). The integrated CD19 or CD22 CAR transgene sequence in patients was detected by quantitative PCR. Genomic DNA was extracted and collected using a QIAamp DNA Blood Mini Kit (Qiagen NV). Quantitative real-time PCR was performed in triplicate in a 7500 real-time PCR system (Thermo Fisher Scientific). Copy numbers per microgram of genomic DNA were calculated from a standard curve with 10 -fold serial dilutions of purified CAR plasmid containing $10^{5}-10^{8}$ copies $/ \mathrm{mL}$. Amplification of an internal control gene was conducted to normalize the DNA quantities. We used the SYBR Green (Vazyme Biotech Co., Ltd., Nanjing, China) to detect the anti-CD19 or anti-CD22 CAR transgene with the application of Woodchuck hepatitis virus posttranscriptional regulatory element (WPRE) and an internal control gene as described previously. ${ }^{12}$

\section{Discussion}

There is no standard protocol for subsequent treatment after recurrence following CD19-modified CAR-T cell therapy for B-ALL, and second infusion with the same CAR-T cells was ineffective because most anti-CD19 CARs carried scFvs derived from murine antibodies. Patients may exhibit activated $\mathrm{T}$-cell immune reactivity against the fragment encoded by the CAR transgene after the first infusion and thus trigger rapid decreases in CAR-T cells mediated by immune rejecting reaction after the second infusion of the same CAR-T cells. ${ }^{9}$ Under this condition, reinfusion of CAR-T cells harboring a humanized $\mathrm{ScFv}$ may induce a second remission.

The patient initially relapsed very quickly. The B cells returned to normal levels. This may be because mousederived CAR-T was ineffective, the tumor cell antigen was mutated but undetected, and the CAR-T cells could not recognize leukemic cells. Therefore, we chose other recognition sites on CAR-CD19 T cells and CAR-CD22 $\mathrm{T}$ cells. Although mutations in the tumor cell antigen were not detected, replacement of CAR-T cells was effective, and the antigen epitope was changed to some extent. The original CAR was not recognized, and thus, the copy number could not be stimulated by the tumor antigen and may change the CAR to have different structures.

CD22 is another marker expressed in most B-ALL cases. ${ }^{13}$ In our case, CD22 was not expressed when the disease diagnosis was established but was expressed when recurrence occurred after infusion with CD19-targeted CAR-T cells. Considering the simultaneous expression of CD19 and CD22, we sequentially infused two T-cell products: T cells with CD19-modified CAR and T cells with anti-CD22 CAR. Sequential infusion of $\mathrm{T}$ cells with CD19-directed CAR 
and $\mathrm{T}$ cells with CD22-targeted CAR for treating B-ALL is advantageous because the antigens are nearly exclusively expressed by cells of the B-cell lineage; the risk of on-target off-leukemia toxicity is low. Because CD19 and CD22 are involved in promoting B-cell survival, ${ }^{13,14}$ the probability of simultaneous loss of both antigens is also low, making mixed targeting a powerful method for reducing the survival of leukemic cells that lost one of the antigens.

\section{Conclusion}

Our case demonstrated the efficacy of sequential infusion of humanized CD19-modified and CD22-modified CAR-T cells for treating relapsed ALL previously exposed to murinederived CD19-targeted CAR-T cells. Further well-designed and randomized studies are needed to fully evaluate this strategy.

\section{Compliance with ethical standards Ethical approval}

The study was approved by the Institutional Review Board of the First Affiliated Hospital of Soochow University.

\section{Informed consent}

Written informed consent was obtained from the patient's father for study entry and publication of this study.

\section{Acknowledgment}

This work was jointly supported by the Project of Invigorating Health Care through Science, Technology and Education, Jiangsu Provincial Key Medical Center (YXZXA2016002), the Priority Academic Program Development of Jiangsu Higher Education Institutions (PAPD) and National Natural Science Foundation of Jiangsu Province (BE2018652).

\section{Disclosure}

The authors report no conflicts of interest in this work.

\section{References}

1. Rosenbaum L. Tragedy, perseverance, and chance - the story of: CAR-T therapy. NEngl J Med.2017;377(14):1313-1315. doi:10.1056/ NEJMp1711886

2. Locatelli F, Schrappe M, Bernardo ME, Rutella S. How I treat relapsed childhood acute lymphoblastic leukemia. Blood. 2012;120(14): 2807-2816. doi:10.1182/blood-2012-02-265884

3. Pulte D, Jansen L, Gondos A, et al. Survival of adults with acute lymphoblastic leukemia in Germany and the United States. PLoS One. 2014;9(1):e85554. doi:10.1371/journal.pone.0085554

4. Grupp SA, Kalos M, Barrett D, et al. Chimeric antigen receptor-modified $\mathrm{T}$ cells for acute lymphoid leukemia. $N$ Engl J Med. 2013;368(16): 1509-1518. doi:10.1056/NEJMoa1215134

5. Maude SL, Frey N, Shaw PA, et al. Chimeric antigen receptor T cells for sustained remissions in leukemia. N Engl J Med. 2014;371(16): 1507-1517. doi:10.1056/NEJMoa1407222

6. Brentjens RJ, Davila ML, Riviere I, et al. CD19-targeted T cells rapidly induce molecular remissions in adults with chemotherapy-refractory acute lymphoblastic leukemia. Sci Transl Med. 2013;5(177):177ra38. doi:10.1126/scitranslmed.3005930

7. Davila ML, Riviere I, Wang X, et al. Efficacy and toxicity management of 19-28z CAR T cell therapy in B cell acute lymphoblastic leukemia Sci Transl Med. 2014;6(224):224ra25. doi:10.1126/scitranslmed. 3008226

8. Lee DW, Kochenderfer JN, Stetler-Stevenson M, et al. T cells expressing CD19 chimeric antigen receptors for acute lymphoblastic leukaemia in children and young adults: a phase 1 dose-escalation trial. Lancet. 2015;385(9967):517-528. doi:10.1016/S0140-6736(14)61403-3

9. Turtle CJ, Hanafi LA, Berger C, et al. CD19 CAR-T cells of defined CD4+: CD8+ composition in adult B cell ALL patients. J Clin Invest. 2016;126(6):2123-2138. doi:10.1172/JCI85309

10. Fry TJ, Shah NN, Orentas RJ, et al. CD22-targeted CAR T cells induce remission in B-ALL that is naive or resistant to CD19-targeted CAR immunotherapy. Nat Med. 2017;24(1):20-28. doi:10.1038/nm.4441

11. Cheadle EJ, Hawkins RE, Batha H, O'Neill AL, Dovedi SJ, Gilham DE. Natural expression of the CD19 antigen impacts the long-term engraftment but not antitumor activity of CD19-specific engineered T cells J Immunol. 2010;184(4):1885-1896. doi:10.4049/jimmunol.0901440

12. Kalos M, Levine BL, Porter DL, et al. T cells with chimeric antigen receptors have potent antitumor effects and can establish memory in patients with advanced leukemia. Sci Transl Med. 2011;3(95):95ra73. doi:10.1126/scitranslmed.3002842

13. Haso W, Lee DW, Shah NN, et al. Anti-CD22-chimeric antigen receptors targeting B-cell precursor acute lymphoblastic leukemia. Blood. 2013;121(7):1165-1174. doi:10.1182/blood-2012-06-438002

14. Otero DC, Rickert RC. CD19 function in early and late B cell development. II. CD19 facilitates the pro-B/pre-B transition. J Immunol. 2003;171(11):5921-5930.
OncoTargets and Therapy

\section{Publish your work in this journal}

OncoTargets and Therapy is an international, peer-reviewed, open access journal focusing on the pathological basis of all cancers, potential targets for therapy and treatment protocols employed to improve the management of cancer patients. The journal also focuses on the impact of management programs and new therapeutic agents and protocols on

\section{Dovepress}

patient perspectives such as quality of life, adherence and satisfaction. The manuscript management system is completely online and includes a very quick and fair peer-review system, which is all easy to use. Visit http://www.dovepress.com/testimonials.php to read real quotes from published authors. 